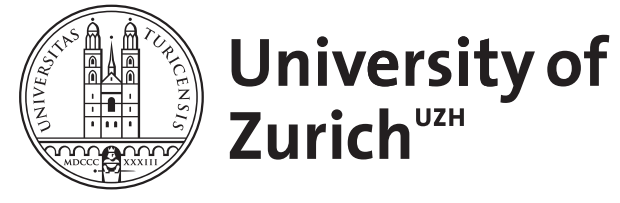

\title{
Multispektrales Kamerasystem für Echtfarben Reproduktion
}

Israel, G ; Niederer, P

DOI: https://doi.org/10.1515/bmte.1996.41.s1.482

Posted at the Zurich Open Repository and Archive, University of Zurich ZORA URL: https://doi.org/10.5167/uzh-154608

Journal Article

Published Version

Originally published at:

Israel, G; Niederer, P (1996). Multispektrales Kamerasystem für Echtfarben Reproduktion. Biomedizinische Technik. Biomedical engineering, 41(s1):482-483.

DOI: https://doi.org/10.1515/bmte.1996.41.s1.482 


\title{
Multispektrales Kamerasystem für Echtfarben Reproduktion
}

\author{
Israel G., Niederer P. \\ Institut für Biomedizinische Technik und Medizinische Informatik \\ ETH und Universität Zürich, 8044 Zürich, Schweiz
}

\section{EINLEITUNG:}

Durch die Verwendung eines multispektralen hochauflösenden digitalen Kamerasystems eröffnet sich der Medizin neu die Möglichkeit des quantitativen Farbvergleichs beispielsweise erkrankter und gesunder Körperpartien verschiedener Patienten. Damit dieser quantitative Farbvergleich von digitalen Farbbildern möglich wird, ist es nötig eine Farbkalibration des Kamerasystems einzuführen, das eine konstante Farbcharakterisierung unabhängig von der Beleuchtung ermöglicht.

\section{METHODE:}

Die Annahme, dass der menschliche Sehapparat spektrale Reize in ein Farbgefühl umwandelt, das durch drei Variablen dargestellt werden kann, ist allgemein akzeptiert [1]. Basierend auf dieser Annahme wurden die "colour-matching functions" (XZY) für den CIE 1931 "Standard Colorimetric Observer" experimentell ermittelt.

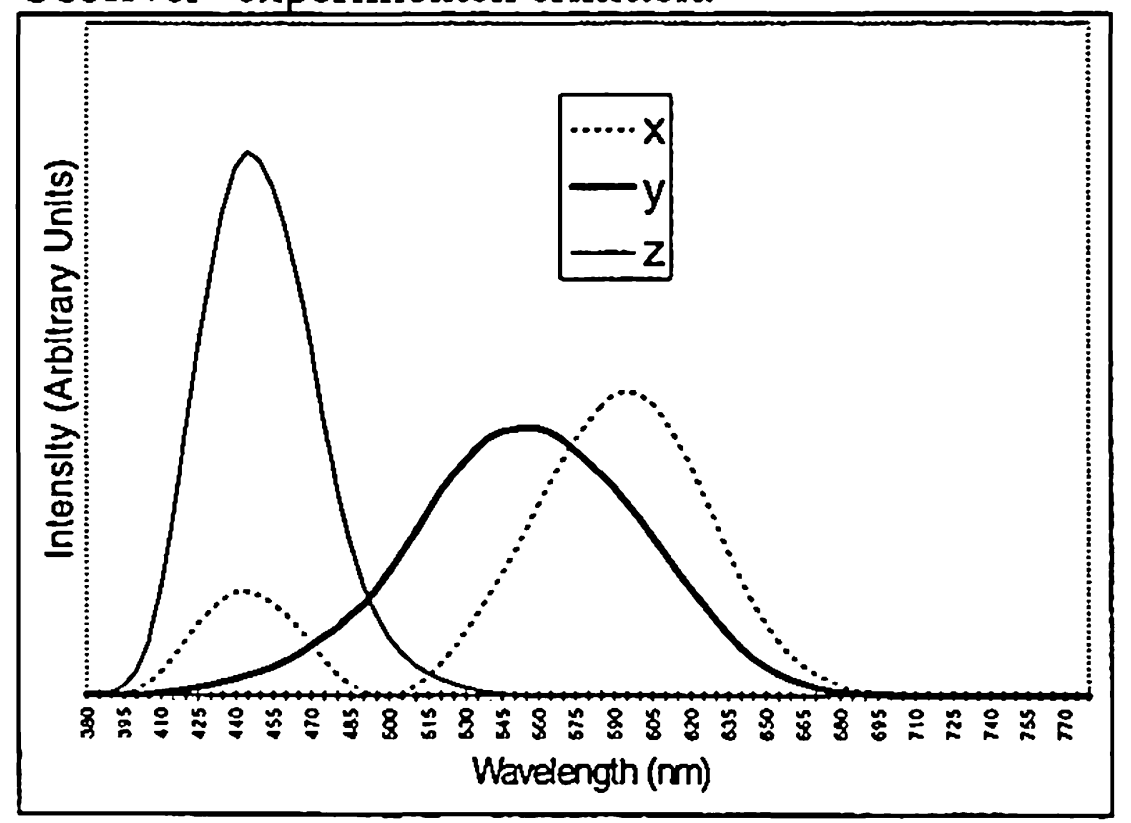

Figur 1: CIE colour-matching functions

Das Integral über die jeweilige Gewichtungsfunktion multipliziert mit dem von einem Körper emittierten Energie Spektrum, liefert eine genaue Farbspezifikation gemäß dem CIE standard Observer. Jedes Farbkamera- System das dieser Farbspezifikation genügen will, muß daher über drei Farbkanäle verfügen, deren spektrale Eigenschaften dem CIE standard Observer entsprechen.

Heute verwendete digital bildgebende Systeme benutzen jedoch nur unzureichend angepasste Farbfilter. Aus technischen Gründen sind gegenwärtig optische Filter welche den colour- matching function genau angepasst sind nicht zu realisieren. Die Akquisition schmalbandiger Bilder und die anschließende Verrechnung der spektralen Teilbereiche gemäss den colour-matching functions bietet sich hier als Alternative an.

Aus diesem Grund wurde ein multispektrales Kamerasystem entwickelt, mit dem es möglich wird, digitalisierte Bilder mit einer durchschnittlichen Bandbreite von 20nm aufzunehmen. Zur Erreichung einer möglichst hohen Bildqualität wurde eine digitale schwarzweiß Videokamera verwendet (siehe Beitrag von A. von Orelli "Hochauflösendes Echtfarben Video System für minimal invasive Applikationen"). Diese Videokamera hat eine Auflösung von 1024*1024 Bildpunkten und eine Dynamik von 60dB (10bit) bei maximal 30 Bildern pro Sekunde. Die hohe Bildqualität dieser Videokamera wird unter anderem durch einen temperaturstabilisierten CCD Sensor erreicht. Durch die Temperaturstabilisation wird der Dunkelstrom des CCD Sensors optimal konstant gehalten, wodurch eine nachträgliche Korrektur von Verstärkung und Offset für jede einzelne Pixel möglich wird. Im Objektiv des Kamerasystems befindet sich ein elektronisch verstellbarer doppelbrechender Filter (LCD, Cambridge Research \& Instruments Inc.). Der Durchlassbereich dieses Filters, läßt sich im Gebiet von $400 \mathrm{~nm}$ bis $720 \mathrm{~nm}$ mit einer durchschnittlichen Bandbreite von 20nm beliebig einstellen.

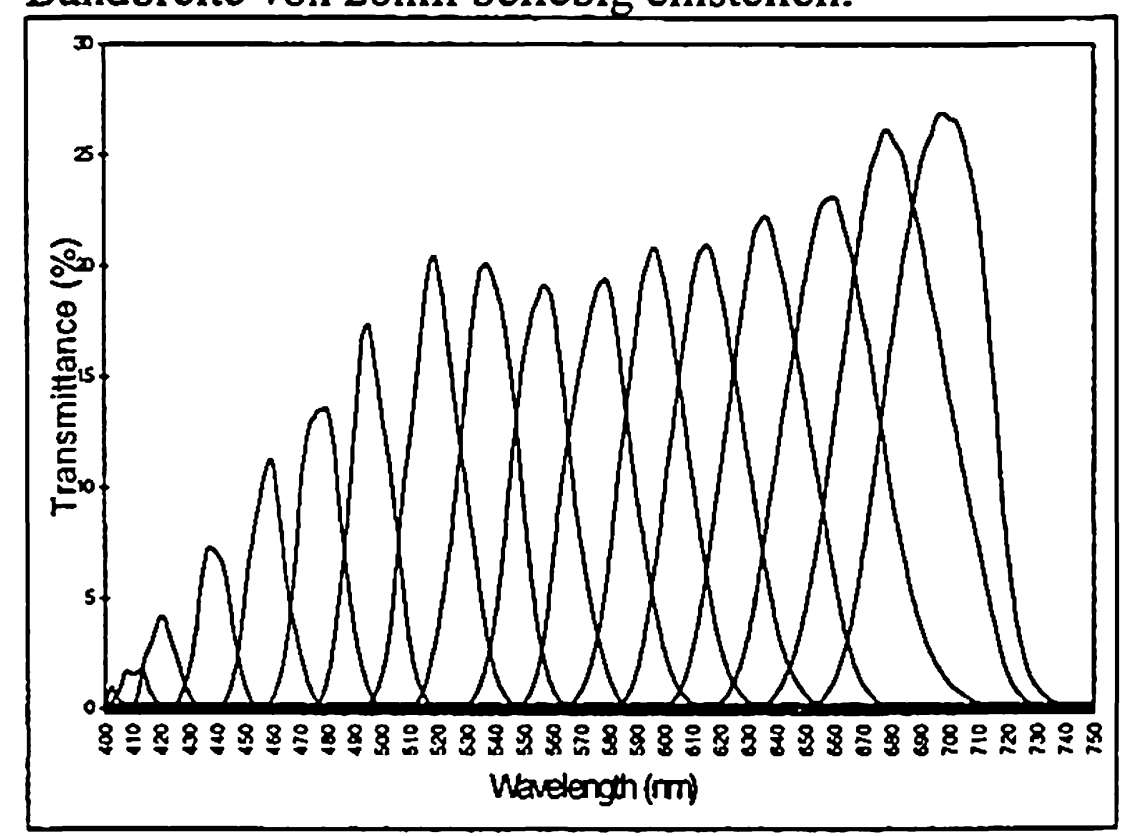

Figur 2: LCD Durchlassbereich

Dieser Filter benötigt etwa $40 \mathrm{~ms}$ für den Umschaltprozess zwischen zwei Einstellungen. Während dieser Umschaltzeit sind die optischen Eigenschaften des Filters nicht definiert, wodurch sich eine Totzeit ergibt, während der keine Bilder 
akquiriert werden können. Weitere $40 \mathrm{~ms}$ werden anschließend als minimale Belichtungszeit für den CCD Sensor benötigt. Hierdurch ergibt sich eine minimale Akquisitionszeit von $80 \mathrm{~ms}$ pro Band, woraus sich eine minimale Gesamtaquisitionszeit für ein 16 bandiges multispektrales Bild von $1.28 \mathrm{~s}$ ergibt. Da die Lichtquanteneffizienz des CCD Sensors im blauen Bereich abnimmt und zugleich die Dämpfung des LCD Filters im gleichen Bereich zunimmt, ist es geplant, die Belichtungszeit des CCD Sensors während jedes Bandes so anzupassen, dass die Dynamik der CCD Kamera immer optimal ausgenutzt wird.

\section{ERGEBNISSE:}

Das Kamerasystems wurde mit einer HalogenBeleuchtungsquelle (MK2, Volpi AG Schlieren) getestet. Das Emissionsspektrum dieser Beleuchtungsquelle samt Lichtleiterbündel, das zur Beleuchtung benutzt wurde, ist in Figur 3 abgebildet. Mit diesem Aufbau wurde eine quasi ideale weisse Kachel mit bekanntem Absorbtionsspektrum beleuchtet, und mit dem multispektralen Kamerasystem aufgenommen. Anschließend wurde die Bildintensität für jedes der Bänder gemessen. Hieraus ergibt sich der in Figur 3 abgebildete spektrale Intensitätsverlauf für ein weisses Objekt unter Verwendung der oben beschriebenen Lichtquelle.

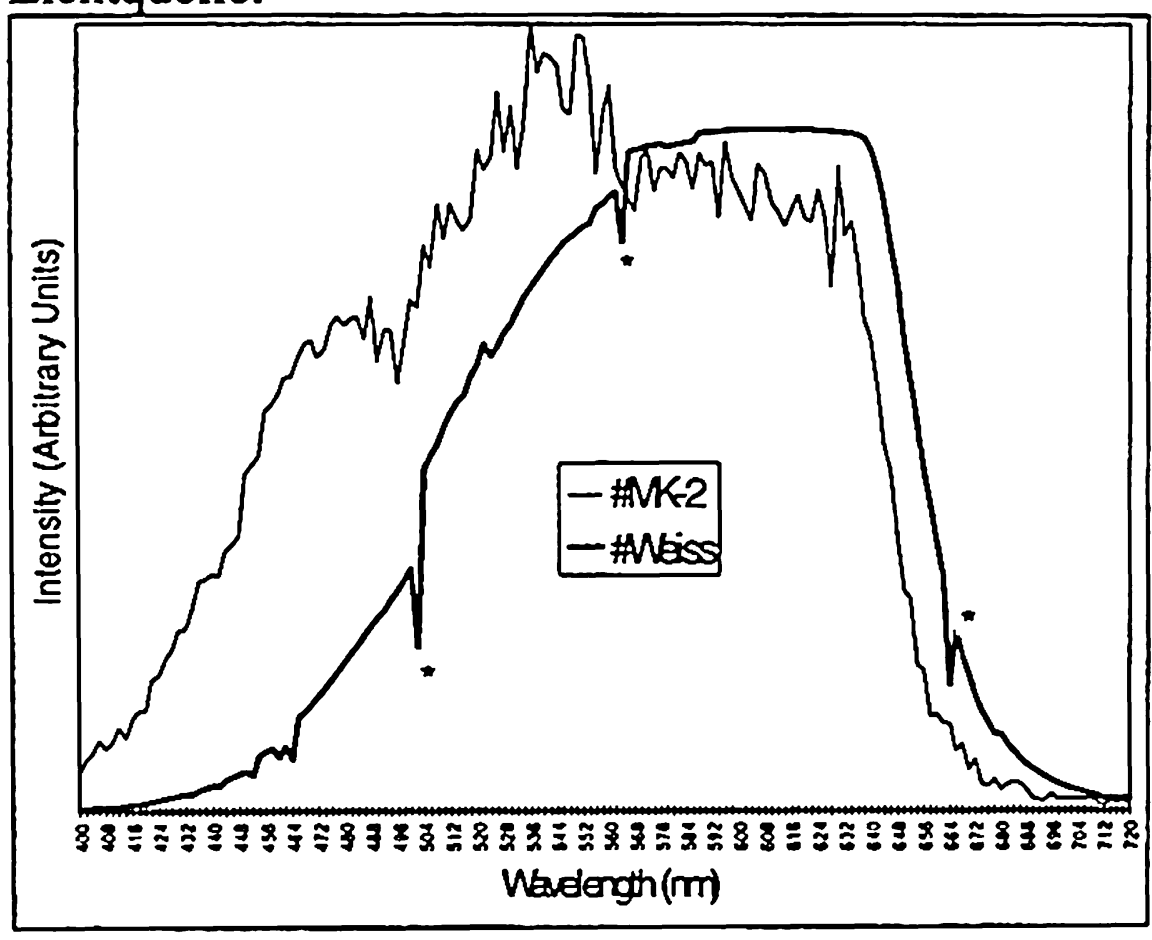

Figur 3: Spektrale Intensität der Beleuchtung und aufgenommene Intensität einer Weisskachel. (Die fett ausgezogene Kurve entspricht der Konvolution des spektralen Verlauf der Lichtquelle und des jeweiligen Durchlassbereiches des LCD Filters. Die durch * gekennzeichneten Artefakte sind auf Bereichänderungen im LCD zurückzuführen)

Es ist nun möglich anhand dieses spektralen Intensitätsverlaufes das gesamte System inklusive Beleuchtung $\mathrm{zu}$ kalibrieren, da das
Absorptionsspektrum der Weisskachel a priori bekannt ist. Bei der Kalibration wird ein Korrekturfaktor für jedes Band berechnet, so dass dessen Intensität auf eine virtuelle Beleuchtung normiert wird (CIE Illuminant E). Hierdurch wird eine Weiss-Referenz für weitere Aufnahmen erzielt

\section{DISKUSSION:}

Wie aus Figur 3 ersichtlich ist, werden bei dem oben beschriebenen Aufbau die Bänder in den Randbereichen des aufgenommenen Spektrums nur ungenügend ausgesteuert. Daraus ergeben sich sehr grosse Korrekturfaktoren für diese Bereiche, was in einem schlechten Signal zu Rausch Verhältnis resultiert. Durch die Verwendung einer angepassten Beleuchtungsquelle, die mehr Energie im blauen Bereich des Spektrums emittiert, z.B. einer Hochdruck Xe Lampe, erhält man eine verbesserte Anpassung der spektralen Empfindlichkeit.

Die hier vorgestellte Methode der Multispektralen Bildgebung schafft die Voraussetzung für eine sehr genaue Farbcharakterisierung, was einen qualitativen Farbvergleich verschiedener Bilder ermöglicht. Eine erste Anwendung bezieht sich auf die Charakterisierung von Melanomen.

\section{LITERATUR:}

[1] R.W.G. Hunt et al.; Measuring Colour, 1991, ISBN 0-13-5676860-X 